\title{
Biaxial 360-degree scanning LIDAR using a liquid crystal control
}

\author{
Seiji Nishiwaki
}

Sophisticated non-mechanical technology for LIDARs is needed to realize safe autonomous cars. We have confirmed the operating principle of a non-mechanical LIDAR by combining concentric circulargrating couplers (CGCs) with a coaxially aligned rod lens. Laser light incident vertically on the center of the inner CGC along the center axis of the lens is radiated from the outer CGC and passes through the side surface of the lens. It is converted to a parallel beam that scans in two axes by applying voltages to two area-segmented electrode layers sandwiching the CGCs and a liquid crystal layer formed on the CGCs. We have demonstrated scanning whose motion ranges were 360 degrees horizontally and $10^{\circ}$ vertically. A beam with a spread angle of $0.3^{\circ} \times 0.8^{\circ}$ at a minimum swept vertically up to a frequency of $100 \mathrm{~Hz}$ and ten equally spaced beams scanned rotationally with a 6-degree cycle variation of spread of between $0.8^{\circ}$ and $3.5^{\circ}$.

Aiming for completion in the next few years, auto manufacturers and information equipment manufacturers across the globe are advancing the development of fully autonomous cars. To realize this "level 5," real-time measurement technology of distance between cars and physical objects is necessary. LIDAR (Light detection and ranging) apparatus using time of flight (TOF) technology is one potential solution. Conventional LIDARs are composed of a mechanical structure ${ }^{1-3}$ such as a polygon mirror or a galvanic mirror mounted on a rotating plate. The downside of this type is that they are expensive, slow, large, heavy, and non-durable due to having moving parts. To overcome these drawbacks, various non-mechanical methods (e.g., solid state LIDAR or SSLIDAR) have been proposed.

A MEMS (microelectromechanical system) mirror ${ }^{4-7}$ or an optical phased array ${ }^{8-16}$ (OPA) can be used for SS-LIDAR. A MEMS mirror is fabricated by etching a silicon crystal and forming a coil structure. The direction of a light ray incident on the mirror surface can be controlled by the Lorentz force produced by energization of the coil. MEMS-LIDAR is likely the most advanced non-mechanical type, but it faces problems with durability because it still has moving parts. Moreover, it is difficult to steer it free from hysteresis caused by creep and it is also easily affected by automotive vibrations due to the use of a resonant mode (non-linear mode) for scanning in the horizontal direction.

For the OPA, although phased arrays are practically realized in the field of radio wave technology, manufacturing challenges remain with the fine-pitched fabrication process that is required. It is also difficult to control the phases emitted from each array and to correct for environmental changes during use.

Another proposition provides a good light sweeper by using a slow-light waveguide ${ }^{17-19}$, in which light is transported with the slow group velocity in multiple layers or photonics crystals. However, few slow-light methods appear to be suitable for commercial use because their beam steering that exploits wavelength changes requires an expensive light source.

Some electro-optic structures ${ }^{20,21}$ and photonic-crystal lasers ${ }^{22,23}$ give provide a good light scanner, but they are limited to one-dimensional steering.

All the above-mentioned SS-LIDARs significantly narrow their steering ranges to realize non-mechanical scanning and most of them (except for MEMS-LIDARs) need detection structures that are physically separate from their emission structures. To provide a robust answer to these challenges, we devised a novel structure (named CGC-LIDAR) using an LC (liquid crystal) and demonstrated the light-scanning principle over a 360-degree sweeping range.

Structure and operational principle of a CGC-LIDAR. Figure $1 \mathrm{a}, \mathrm{b}$ are a perspective and cross-sectional illustration of a CGC-LIDAR that includes concentric grating couplers (CGCs) ${ }^{24-26}$. Light of wavelength $\lambda=0.94 \mu \mathrm{m}$ emitted from a laser diode is collimated to parallel light by a collimator and is reflected to a polarizing beam splitter (PBS). It is then focused by a focal lens on the inner CGC, passing through a 1/4 wave plate, a half mirror, and the rod lens along axis L. The circularly polarized light vertically incident on the CGC excites 


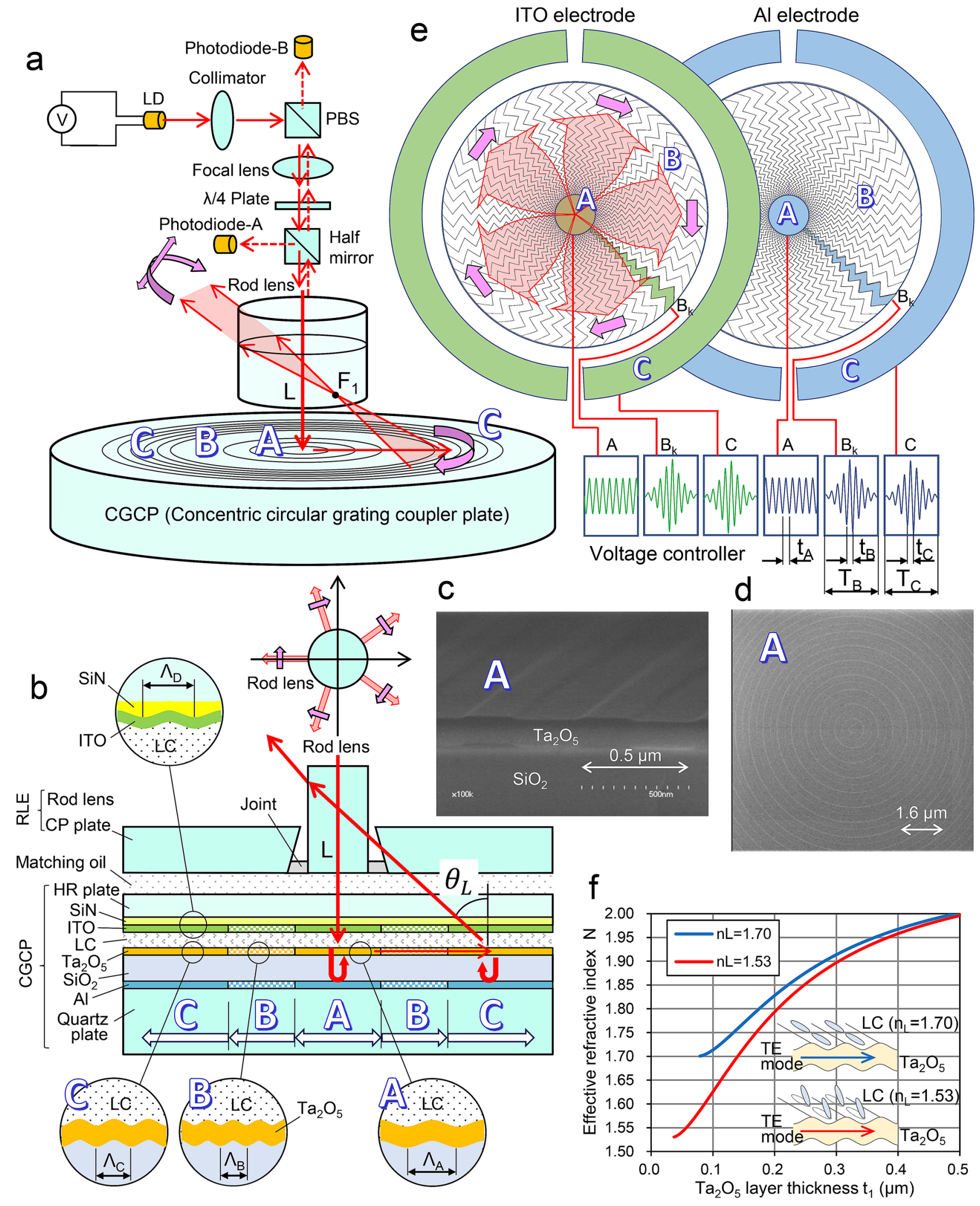


४Figure 1. Structure and operational principles of CGC-LIDAR. (a) Perspective illustration of a CGC-LIDAR. The circularly polarized light vertically incident to coupler A excites a guided light which propagates uniformly from the center to outside. The guided light is radiated from coupler $\mathrm{C}$, concentrated near point $\mathrm{F}_{1}$, and converted to 2D-scannable five parallel beams by refraction at the side surfaces of the rod lens. (b) Crosssectional illustration of a CGC-LIDAR. Concentric circular gratings are formed on the $\mathrm{Ta}_{2} \mathrm{O}_{5} / \mathrm{SiO}_{2}$ layers of the quartz plate and on the ITO/SiN layers of the HR plate. The upper and lower gratings and electrodes are divided from the inside to outside into the three areas of A, B, and C. The LC layer sandwiched by the two plates is homogeneously aligned along the rotational direction around the axis $\mathrm{L}$ due to the orientation force of the gratings. CGC-LIDAR is built up concentrically with an RLE and a CGCP which are joined with a matching oil. (c) Perspective-view SEM photographs of coupler A. (d) Top-view SEM photograph of coupler A. (e) Configuration diagram of electrodes for areas A, B, and C. The electrode B is divided into 60 areas of $B_{k}(k=0$ to 59) in the rotational direction. While the electrodes $A$ and $C$, shaped as circles or half rings, have the same shapes between the Al layer and the ITO layer, the $B_{k}$ electrodes with a zigzag shape are reversed between them. The electrode $\mathrm{C}$ is divided in half and the gaps between them are used for the wires of electrodes $\mathrm{A}$ and $\mathrm{B}_{\mathrm{k}}$. Since the AC signals applied to the electrodes are reversed between the Al electrode and the ITO electrode, the differences in voltages between them are doubled. (f) Relationships between effective refractive index (ERI) and waveguide thickness. When AC signals are applied to the electrodes, the LC alignment is raised, and ERI falls in inverse proportion to the AC amplitude.

the guided light in TE mode in the waveguide layer which propagates uniformly from the center to outside. The guided light is radiated from the coupler $\mathrm{C}$ and is concentrated near point $\mathrm{F}_{1}$ by $\mathrm{LC}$ control. It is then converted to a parallel beam by refraction at the side surfaces of the rod lens. Five parallel beams are generated equiangularly around the rod lens and are rotated by LC control. Diffused light reflected from an outer object returns to the coupler $\mathrm{C}$ and excites the guided light in the opposite direction. The return light output from the coupler $\mathrm{A}$ is detected by photodiodes A and B. TOF signals are produced by the two detectors. Our method produces multisweep beams in principle, and these beams also produce individual TOF signals. They can be separated by using the two detectors (see Supplementary information S1).

CGC-LIDAR is built up concentrically with an RLE (rod lens element) and a CGCP (CGC plate) which are joined with a matching oil of refractive index 1.59. The RLE consists of a rod lens (radius $\mathrm{r}_{0}=0.95 \mathrm{~mm}$ ) and a CP (circularly perforated) plate, which are built along axis $\mathrm{L}$ and fastened together with a joint that aligns their bottom surfaces (see Supplementary information S2). The CGCP is built of a quartz plate and an HR (high refractive index, index 2.0) plate by encapsulating a $5 \mu$ m-thick LC layer, made of $5 \mathrm{CB}$.

On the LC-side surface of the quartz plate, an aluminum (Al) layer $\left(0.09 \mu \mathrm{m}\right.$ thick), a $\mathrm{SiO}_{2}$ (silicon oxide) layer $\left(\mathrm{t}_{0}=1.16 \mu \mathrm{m}\right.$ thick) and $\mathrm{Ta}_{2} \mathrm{O}_{5}$ (tantalum oxide) layer $\left(\mathrm{t}_{1}=0.132 \mu \mathrm{m}\right.$ thick) are formed sequentially. The $\mathrm{Al}$ layer acts as an electrode (or a reflector) and is divided from inside to outside into three areas: $\mathrm{A}, \mathrm{B}$, and $\mathrm{C}$ (radius $0-0.10 \mathrm{~mm}, 0.30-4.01 \mathrm{~mm}$, and $4.50-7.00 \mathrm{~mm}$ ).

As shown in Fig. 1c,d, concentric circular gratings $(\mathrm{d}=0.013 \mu \mathrm{m}$ deep) divided into three areas of $\mathrm{A}, \mathrm{B}$, and $\mathrm{C}$ (radius $0-0.05 \mathrm{~mm}, 0.05-4.50 \mathrm{~mm}$, and $4.50-6.50 \mathrm{~mm}$ ) are formed concentrically along the axis $\mathrm{L}$ by electron lithography on the $\mathrm{SiO}_{2}$ layer after a planarizing process using CMP (chemical mechanical polishing). The $\mathrm{SiO}_{2}$ layer acts as a buffer layer and the $\mathrm{Ta}_{2} \mathrm{O}_{5}$ layer acts as a waveguide. While the gratings in areas $\mathrm{A}$ and $\mathrm{C}$ behave as grating couplers because their grating pitches $\left(\Lambda_{\mathrm{A}}=0.544\right.$ and $\left.\Lambda_{C}=0.295 \mu \mathrm{m}\right)$ are larger than the critical level of coupling $(0.27 \mu \mathrm{m})$, the grating area $B$ does not behave as a coupler because its pitch $\left(\Lambda_{B}=0.240 \mu \mathrm{m}\right)$ is smaller. The duty rates $\varepsilon$ of the gratings in area A and B are 0.5 , and that in C is 0.2 (see Supplementary information S3).

Whereas, a SiN (silicon nitride) layer $(0.10 \mu \mathrm{m}$ thick) and an ITO (indium tin oxide) layer $(0.10 \mu \mathrm{m}$ thick) are formed on the LC-side surface of the HR plate. Concentric circular gratings $\left(0.05 \mu \mathrm{m}\right.$ deep, period $\left.\Lambda_{\mathrm{D}}=1.2 \mu \mathrm{m}\right)$ are concentrically formed from inside to outside by reduced projection exposure along the axis $\mathrm{L}$ on the surface of the SiN layer. The ITO layer behaves as an electrode, and in the same way as the Al layer, is divided into three areas: $\mathrm{A}, \mathrm{B}$, and $\mathrm{C}$.

As shown in Fig. 1e, the electrode $B$ is further divided into 60 areas of $B_{k}(k=0$ to 59$)$ in the rotational direction. The electrode $\mathrm{C}$ is divided into halves to lace wires to the outside. While electrodes $\mathrm{A}$ and $\mathrm{C}$ have the same shapes between the Al layer and the ITO layer, the $B_{k}$ electrodes, which have a zigzag shape (see Supplementary information S4), are reversed between them. Although the $B_{k}$ electrodes do not need wiring because their shapes are equivalent to wiring, they must use the spaces between electrodes $\mathrm{B}$ and $\mathrm{C}$ (i.e., radius $4.01-4.50 \mathrm{~mm}$ ) to link them to the outside. For electrode $A$, one electrode of $B_{k}$ is used as a wire instead. Rectangular or triangular AC (alternating current) voltages of cycle $t_{A}, t_{B}$, and $t_{C}$, respectively, are applied to electrodes $A, B$, and $C$. While an envelope waveform of constant $\mathrm{AC}$ amplitude is applied to electrode $\mathrm{A}$, varying waveforms are applied to the electrodes of $B_{k}$ and $C$ with cycle $T_{B}$ and $T_{C}$, which are related to beam sweeping cycles in the horizontal and vertical directions. Since the AC signals are reversed between the Al electrode and the ITO electrode, the differences in voltages between them are doubled.

There is no other alignment structure (or surface treatment) than the gratings. Thus, the LC layer sandwiched by the two plates is homogeneously and concentrically aligned in the rotational direction around the axis $\mathrm{L}$ due to the orientation force of the gratings remaining on the surfaces of the $\mathrm{Ta}_{2} \mathrm{O}_{5}$ layer and the ITO layer (see Supplementary information S5). As shown in Fig. 1f, when AC signals are applied to the electrodes, the alignment of the LC molecules is raised in a plane contacting both the thickness direction of the LC and the grating direction. The effective refractive index (ERI) of $\mathrm{N}$ for the guided light declines as a function of increasing AC amplitude due to change of $n_{L}$ (i.e., the refractive index of the LC layer for the guided light of TE mode).

The voltage control of electrode A optimizes the coupling conditions at the coupler A. The voltage of electrode $\mathrm{C}$ controls the vertical scan according to the formula of 

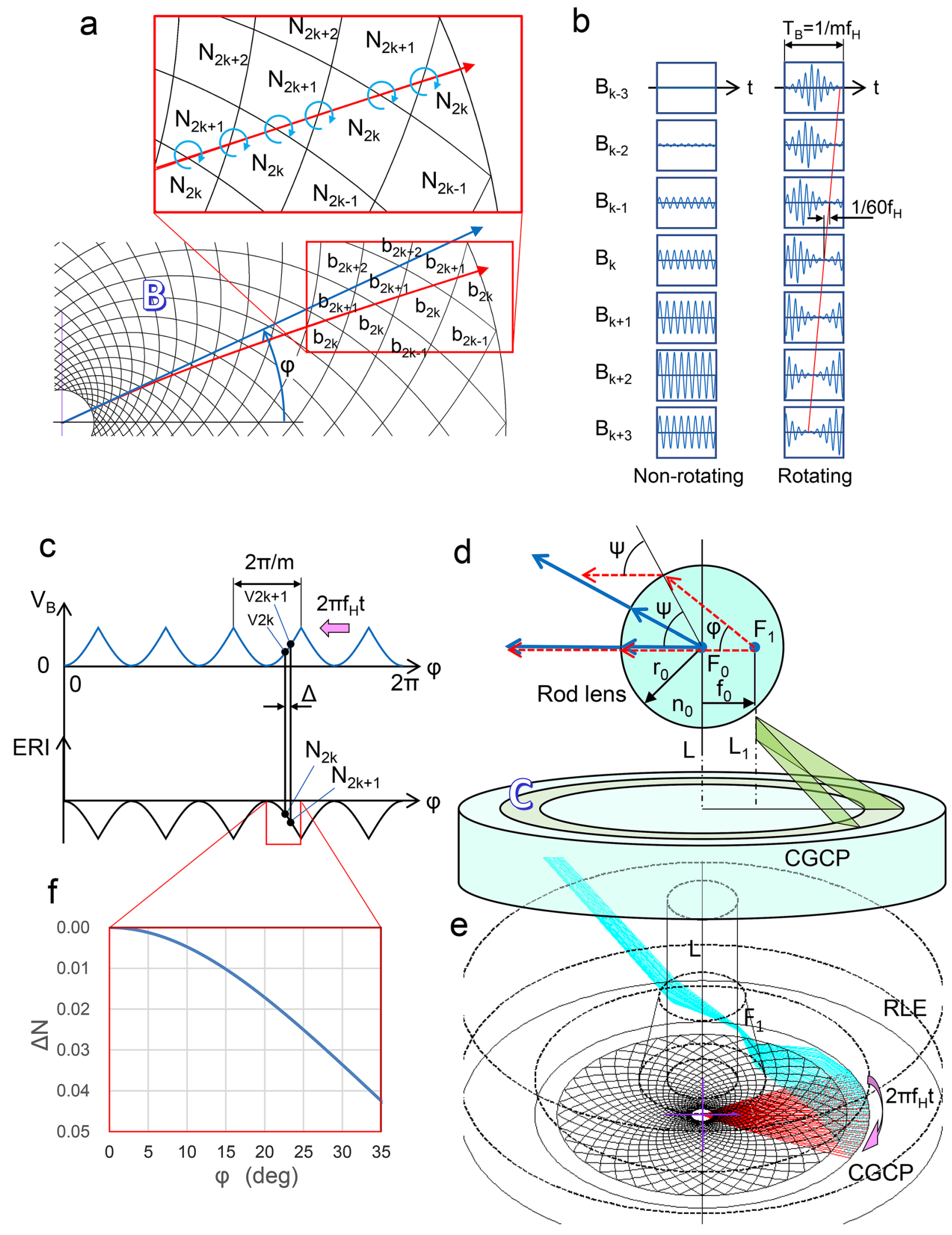
4Figure 2. Beam-condensing and rotating principle in the horizontal direction. (a) Electrode pairs composed of upper and lower electrodes $B_{k}$ and a trace of guided light. Electrodes $b_{2 k}$ are generated from the combination of Al electrodes $B_{k}$ and ITO electrodes $B_{k}$, and electrodes $b_{2 k+1}$ are from Al electrodes $B_{k}$ and ITO electrodes $\mathrm{B}_{\mathrm{k}+1}$. In response to the voltages $\mathrm{v}_{2 \mathrm{k}}$ or $\mathrm{v}_{2 \mathrm{k}+1}$ at electrodes $\mathrm{b}_{2 \mathrm{k}}$ or $\mathrm{b}_{2 \mathrm{k}+1}$, the ERIs of $\mathrm{N}_{2 \mathrm{k}}$ or $\mathrm{N}_{2 \mathrm{k}+1}$ are aligned in the radial direction. The guided light deflects to the higher-index side at the intersection with the boundary of the diamond-shaped electrodes, as shown by the red arrow. (b) Examples of voltage waveforms applied to the $B_{k}$ electrodes. Amplitude distribution between the electrodes $B_{k}$ allows the beams to be collimated. Collimated beams can be rotated by applying a phase-shift signal to the neighbor electrodes. c. Relationships under a static condition between a voltage waveform and the response of the ERI to an azimuth angle $\varphi$. The waveform is represented by a collection of voltage elements $v_{2 k}$ and $v_{2 k+1}$ which are applied to the electrodes $b_{2 k}$ and $b_{2 k+1}$. The curve of ERI traces a shape that is the inverse of waveform $V_{B}$. (d) Perspective illustration of light radiated from the coupler $\mathrm{C}$ and a cross-sectional drawing of light refracted at the rod lens surface. When applied to the $\mathrm{B}_{\mathrm{k}}$ electrodes, the radiated light is focused on point $\mathrm{F}_{1}$ and becomes a parallel beam after refraction at the side surface of the rod lens. (e) Ray-traced result for light that propagates in the guided layer and penetrates the RLE. This is an example for $m=5$. In the range of $\varphi=-36$ to 36 degrees, the guide light is convergent and radiates from the coupler $C$. It becomes a parallel beam after focusing at point $F_{1}$ and passing through the rod lens. This situation is similar in the other angular ranges and five parallel beams are produced in total. (f) Necessary shape of the curve of the ERI difference.

$$
n_{L} \sin \theta_{L}=\lambda / \Lambda_{C}-N,
$$

where $\theta_{\mathrm{L}}$ is the radiation angle in the LC layer (see Supplementary information S2). The waveforms applied to the $B_{k}$ electrodes adjust the focusing position $F_{1}$ to convert the radiated light to five parallel beams (see Supplementary information S6). Rotation of this waveform produces a horizontal scan around the axis $\mathrm{L}$.

Beam-collimating and rotating principle in the horizontal direction. Figure $2 \mathrm{a}$ shows a portion of an electrode pair composed of the ITO and $\mathrm{Al}$ electrode, in which vertexes of zigzag shapes between the two electrodes of $B_{k}$ overlap together and diamond shape electrodes such as $b_{2 k}$ and $b_{2 k+1}$ are aligned in the radial direction, where $b_{2 k}$ are generated from the combination of Al electrodes $B_{k}$ and ITO electrodes $B_{k}$, and $b_{2 k+1}$ are from $\mathrm{Al}$ electrodes $\mathrm{B}_{\mathrm{k}}$ and ITO electrodes $\mathrm{B}_{\mathrm{k}+1}$. The voltage amplitude at the electrodes $\mathrm{b}_{2 \mathrm{k}+1}$ becomes the average between those at the electrodes $b_{2 k}$ and the electrodes $b_{2 k+2}$. When the AC signals are applied to the electrodes of $\mathrm{B}_{\mathrm{k}}$, the ERIs of such as $\mathrm{N}_{2 \mathrm{k}}$ or $\mathrm{N}_{2 \mathrm{k}+1}$, in response to the voltages $\mathrm{v}_{2 \mathrm{k}}$ or $\mathrm{v}_{2 \mathrm{k}+1}$ at electrodes $\mathrm{b}_{2 \mathrm{k}}$ or $\mathrm{b}_{2 \mathrm{k}+1}$, are aligned in the radial direction.

Figure $2 \mathrm{~b}$ show examples of voltage waveforms applied to the $\mathrm{B}_{\mathrm{k}}$ electrodes. For a non-rotating condition (or a static state), the AC waveform envelopes are constant, with their signal amplitudes varying sinusoidally according to the value of $\mathrm{k}$. Amplitude distribution between the electrodes allows the beams to be collimated. Under rotating conditions, the envelopes have a sinusoidal shape and the phase between the neighbor signals shifts together by $1 / 60 \mathrm{f}_{\mathrm{H}}$. Collimated beams can be rotated by applying a phase shift to the sinusoidal amplitude.

Figure $2 c$ shows relationships under a static condition between a voltage waveform $V_{B}$ applied to the $B_{k}$ electrodes and the response of the ERI to an azimuth angle $\varphi(=2 \mathrm{k} \pi / 60$ or $(2 \mathrm{k}+1) \pi / 60)$. The waveform $\mathrm{V}_{\mathrm{B}}$ has a periodic number $\mathrm{m}(\mathrm{m}=5$ or 10$)$ per revolution and is represented by a collection of elements of $\mathrm{v}_{2 \mathrm{k}}$ or $\mathrm{v}_{2 \mathrm{k}+1}$. $\mathrm{N}_{2 \mathrm{k}}$ $\left(\right.$ or $\mathrm{N}_{2 \mathrm{k}+1}$ ) is given from $\mathrm{v}_{2 \mathrm{k}}\left(\right.$ or $\mathrm{v}_{2 \mathrm{k}+1}$ ) and its curve traces a shape that is the inverse of waveform $\mathrm{V}_{\mathrm{B}}$. If $\mathrm{N}_{2 \mathrm{k}}>\mathrm{N}_{2 \mathrm{k}+1}$ as shown in Fig. 2 c, the guided light (shown by the red arrow in Fig. 2a) deflects to the higher index side (in the direction of the blue circling arrows) at the intersection with the boundary of the diamond-shaped electrodes. The differences in indexes are small. However, since the guided light passes through more than 30 boundaries within the range of area B, the accumulated deflection angle of the red arrow eventually totals about 10 degrees in comparison with the blue and straight arrow. This deflecting principle is similar to that of an electro-optic scanner ${ }^{20}$. The rotating condition corresponds to the movement of the waveform at a frequency $\mathrm{f}_{\mathrm{H}}$ as indicated by an arrow shown in Fig. 2c (see information S7).

Figure $2 \mathrm{~d}$ shows a combination of a perspective illustration of light radiated from the coupler $\mathrm{C}$ and a crosssectional drawing of light refracted at the rod lens surface. Figure $2 \mathrm{e}$ shows a ray-tracing result for light that propagates in the guided layer, is then radiated from the coupler $\mathrm{C}$, and penetrates RLE. If no difference in voltage is applied to the $B_{k}$ electrodes, the guided light propagates in the direction of the blue arrow in Fig. 2a: the radiated light is focused at point $\mathrm{F}_{0}$ on the center axis $\mathrm{L}$ of the rod lens and travels in a straight line in the radial direction as shown by the blue arrows in Fig. $2 \mathrm{~d}$. In this case, the radiated light becomes a widely spread beam with a conical wavefront. When applied to the $B_{k}$ electrodes, the guided light propagates in the direction of the red arrow in Fig. 2a and the radiated light is focused on point $F_{1}$, which moves the distance of $f_{0}$ from $F_{0}$. The light becomes a parallel beam after refraction at the side surface of the rod lens, as shown by the red dotted arrows. This beam is generated by the period number $\mathrm{m}$ of the waveform, that is, five or ten. To correct the aberration of the radiated beams (see Supplementary information S8), the light focused on $\mathrm{F}_{1}$ needs a phase difference of $n_{0} f_{0}(1-\cos \varphi)$,where

$$
f_{0}=\frac{r_{0} \sin \psi}{n_{0} \sin \varphi}, \quad \sin \psi=\frac{n_{0} \sin \varphi \sqrt{n_{0}^{2}+1+2 n_{0} \cos \varphi}}{\sqrt{\left(n_{0}^{2}-1\right)^{2}+4 n_{0}^{2} \sin ^{2} \varphi}}
$$



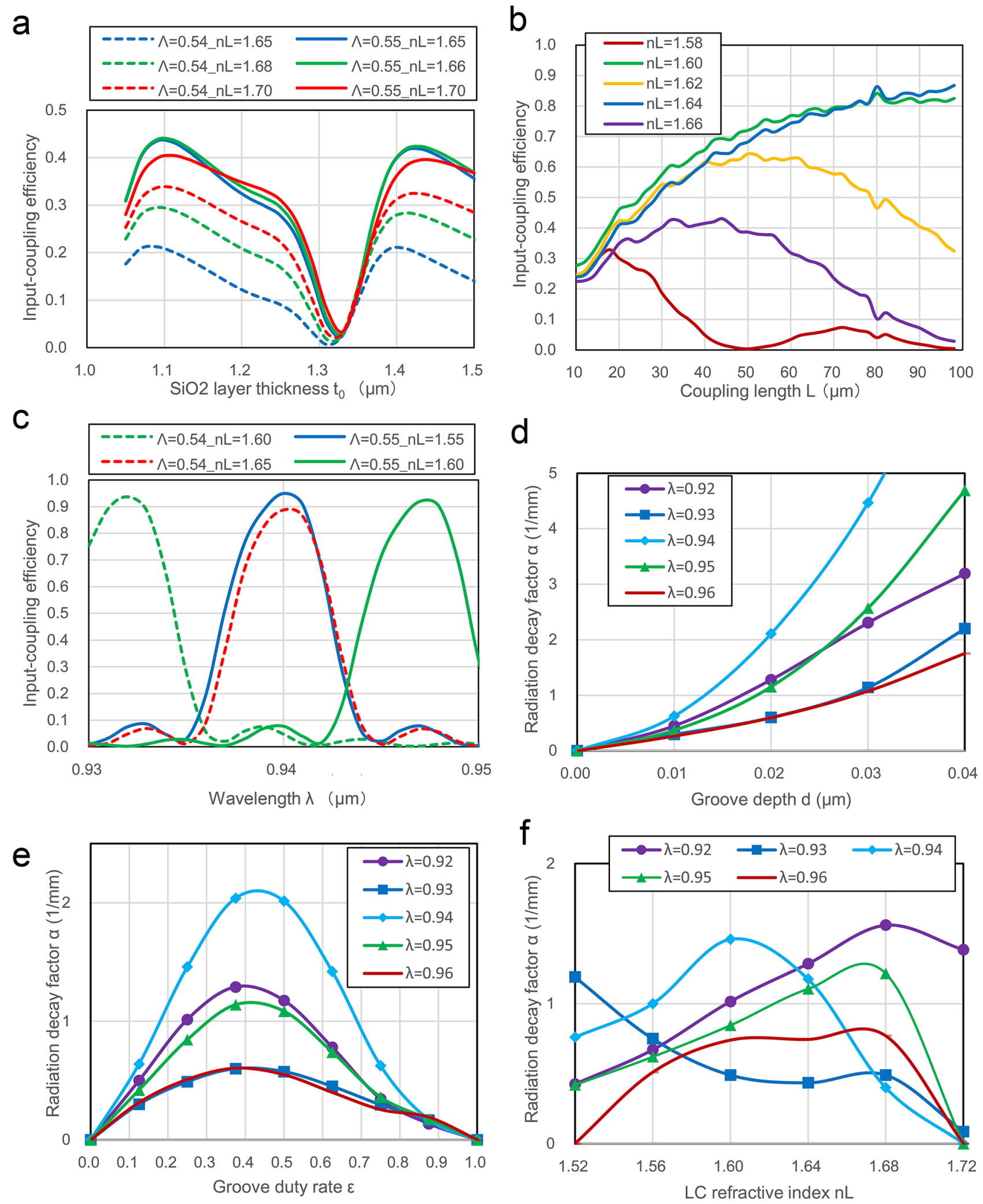
4Figure 3. Analytical results for input- and output-coupling characteristics. (a) Response of input-coupling efficiency at coupler $A$ to a buffer layer thickness $t_{0}$, where a circularly polarized Gaussian light is vertically incident. This is calculated by 3D-FDTD for $\lambda=0.94 \mu \mathrm{m}, \Lambda=0.54$ and $0.55 \mu \mathrm{m}, \mathrm{t}_{1}=0.14 \mu \mathrm{m}$, groove depth $\mathrm{d}=0.1 \mu \mathrm{m}$, groove duty rate $\varepsilon=0.5$, and diameter $\mathrm{L}=10 \mu \mathrm{m}: \mathrm{t}_{0}=1.16 \mu \mathrm{m}$ is appropriate for counteracting film thickness error. (b) Responses of input-coupling efficiency at coupler A to coupling length $\mathrm{L}$ where an S-polarized light is vertically incident. This is calculated by $2 \mathrm{D}$-FDTD for $\lambda=0.94 \mu \mathrm{m}, \Lambda=0.55 \mu \mathrm{m}, \mathrm{t}_{1}=0.14 \mu \mathrm{m}$, $\mathrm{t}_{0}=1.16 \mu \mathrm{m}, \mathrm{d}=0.02 \mu \mathrm{m}$, and $\varepsilon=0.5$. (c) Responses of input-coupling efficiency at coupler A to wavelength $\lambda$ where an S-polarized light is vertically incident. This is calculated by $2 \mathrm{D}$-FDTD for $\Lambda=0.54$ and $0.55 \mu \mathrm{m}$, $\mathrm{d}=0.02 \mu \mathrm{m}, \varepsilon=0.5, \mathrm{t}_{1}=0.14 \mu \mathrm{m}, \mathrm{t}_{0}=1.16 \mu \mathrm{m}$, and coupling length $\mathrm{L}=90 \mu \mathrm{m}$. (d-f) Responses of radiation decay factor $\alpha$ for TE mode light at coupler $C$ to groove depth $d$ (where $\varepsilon=0.5$ and $n_{L}=1.60$ ), groove duty rate $\varepsilon$ (where $\mathrm{d}=0.02 \mu \mathrm{m}$ and $\mathrm{n}_{\mathrm{L}}=1.60$ ), and LC index $\mathrm{n}_{\mathrm{L}}$ (where $\mathrm{d}=0.02 \mu \mathrm{m}$ and $\varepsilon=0.25$ ). These are calculated by 2D-FDTD for $\Lambda=0.30 \mu \mathrm{m}, \mathrm{t}_{1}=0.14 \mu \mathrm{m}$, and $\mathrm{t}_{0}=1.16 \mu \mathrm{m}$ : the factor $\alpha$ can be brought below $1 \mathrm{~mm}^{-1}$ by adopting the design condition of $\mathrm{d}=0.02 \mu \mathrm{m}$, and $\varepsilon=0.2$.

Therefore, the difference in ERI caused in the range of the area B (radius $r_{1}$ to $r_{2}$ ) becomes $\Delta N=\frac{n_{0} f_{0}(1-\cos \varphi)}{r_{2}-r_{1}}$, as shown in Fig. $2 \mathrm{f}$.

The focal line $\mathrm{L}_{1}$ generated by the aggregate of point $\mathrm{F}_{1}$ is not actually parallel with the axis $\mathrm{L}$, and slightly lists so as to broaden towards the top with aberrations produced in the horizontal direction. Therefore, to correct those aberrations, an inverted truncated cone is better than a rod shape (see Supplementary information S2).

Analytical results for coupling characteristics and design condition. Coupler A should ideally be shorter because its coupling efficiency becomes insensitive to changes in wavelength and incident angle. Coupler $\mathrm{C}$ needs to be longer because the spread angle of the radiated beam is inversely proportional to the beam width (i.e., coupling length). It is necessary to satisfy these two contradictory conditions by adjusting the coupling length $\mathrm{L}$ and the groove duty rate $\varepsilon$, with the grating depths $\mathrm{d}$ of couplers $\mathrm{A}$ and $\mathrm{C}$ remaining the same because they use the same etching processes.

Figure $3 a-c$ show the responses of input-coupling efficiency at coupler $A$ to the buffer layer thickness $t_{0}$, coupling length $\mathrm{L}$, and wavelength $\lambda$, respectively, with the parameters of the refractive index $\mathrm{n}_{\mathrm{L}}$ of the LC layer, where light is vertically incident. Since 3D-FDTD calculations over side $10 \mu \mathrm{m}$ far exceed available computing capacity, Fig. 3b,c are analyzed in the 2D model where incident light is S-polarized and uniformly distributed, while maintaining the other structural dimensions. Figure $3 \mathrm{a}$ is designed to show to what extent a circular polarized Gaussian beam can be input-coupled to the coupler A in the 3D model while decreasing coupling size, but instead by increasing the groove depth to accurately maintain the input-coupling efficiency.

Figure $3 \mathrm{a}$ is calculated under the condition of $\lambda=0.94 \mu \mathrm{m}, \Lambda=0.54$ and $0.55 \mu \mathrm{m}, \mathrm{t}_{1}=0.14 \mu \mathrm{m}, \mathrm{d}=0.1 \mu \mathrm{m}$, $\varepsilon=0.5$, and diameter $\mathrm{L}=10 \mu \mathrm{m}$. Figure $3 \mathrm{~b}, \mathrm{c}$ result for $\lambda=0.94 \mu \mathrm{m}, \Lambda=0.55 \mu \mathrm{m}, \mathrm{t}_{1}=0.14 \mu \mathrm{m}, \mathrm{t}_{0}=1.16 \mu \mathrm{m}$, $\mathrm{d}=0.02 \mu \mathrm{m}$, and $\varepsilon=0.5$, and for $\Lambda=0.54$ and $0.55 \mu \mathrm{m}, \mathrm{d}=0.02 \mu \mathrm{m}, \varepsilon=0.5, \mathrm{t}_{1}=0.14 \mu \mathrm{m}, \mathrm{t}_{0}=1.16 \mu \mathrm{m}$, and coupling length $\mathrm{L}=90 \mu \mathrm{m}$, respectively.

From Fig. $3 \mathrm{a}$, a coupling efficiency exceeding $40 \%$ is anticipated by controlling the thickness $\mathrm{t}_{0}$, and $\mathrm{t}_{0}=1.16 \mu \mathrm{m}$ appears to be appropriate for counteracting film thickness error. If the coupling length or grating depth of coupler $C$ is sufficient, all the input-coupled light at coupler $A$ is radiated from $C$ and is converted to collimated beams.

From Fig. 3b,c, by controlling the LC index $\mathrm{n}_{\mathrm{L}}$, sufficient efficiency for coupler A can be achieved by the design condition of $\mathrm{d}=0.02 \mu \mathrm{m}$ and $\varepsilon=0.5$, and $\mathrm{L}=90-100 \mu \mathrm{m}$ and $\Lambda=0.54-0.55 \mu \mathrm{m}$ are appropriate for maximizing the coupling efficiency.

Figure $3 \mathrm{~d}-\mathrm{f}$ show responses of a radiation decay factor $\alpha$ for TE mode light at coupler $\mathrm{C}$ to groove depth $\mathrm{d}$ (where $\varepsilon=0.5$ and $\mathrm{n}_{\mathrm{L}}=1.60$ ), groove duty rate $\varepsilon$ (where $\mathrm{d}=0.02 \mu \mathrm{m}$ and $\mathrm{n}_{\mathrm{L}}=1.60$ ), and LC index $\mathrm{n}_{\mathrm{L}}$ (where $\mathrm{d}=0.02 \mu \mathrm{m}$ and $\varepsilon=0.25$ ), respectively, with parameters of a wavelength $\lambda$, calculated by 2D-FDTD for $\Lambda=0.30 \mu \mathrm{m}$, $\mathrm{t}_{1}=0.14 \mu \mathrm{m}$, and $\mathrm{t}_{0}=1.16 \mu \mathrm{m}$. When guided light propagates along a leaky waveguide such as a grating coupler, its light amplitude along the $\mathrm{x}$ axis decays exponentially as a function of $\exp (-\alpha \mathrm{x})$. This coefficient $\alpha$ is defined as the radiation decay factor, which increases with increased $d$ and as $\varepsilon$ approaches $0.5^{27}$. The value of $1 / 2 \alpha$ corresponds to the effective coupling length.

From Fig. 3d-f, to increase the coupling length of coupler $C$, the factor $\alpha$ can be less than $1 \mathrm{~mm}^{-1}$ (that is, the radiation range can exceed $0.5 \mathrm{~mm}$ ) under the design conditions of $\lambda=0.93-0.94 \mu \mathrm{m}, \mathrm{d}=0.02 \mu \mathrm{m}$, and $\varepsilon=0.2$.

Based on the above results, coupler $\mathrm{A}$ is designed under the conditions of $\Lambda=0.544 \mu \mathrm{m}$ (tuned by taking the wavelength error into account), $d=0.02 \mu \mathrm{m}$ (finally fabricated at $0.013 \mu \mathrm{m}$ ), $\varepsilon=0.5$ and radius $50 \mu \mathrm{m}$, and coupler $\mathrm{C}$ is designed under the conditions of $\Lambda=0.295 \mu \mathrm{m}, \varepsilon=0.2$ and $\mathrm{L}=1.5 \mathrm{~mm}$.

\section{Experimental system}

We can observe a scanning beam radiated from the CGCP sample using the experimental system. Figure 4a shows a configuration diagram of the system (see Methods in detail). Figure $4 \mathrm{~b}-\mathrm{d}$ show perspective photographs of a portion of the system, the RLE, and the CGCP. Figure 4e,f, respectively, are micrographs of the surface of the CGCP where the RLE is removed and located.

Light emitted from a DFB-LD (distributed-feedback laser diode, $\lambda=0.936 \mu \mathrm{m}$ measured) is collimated and vertically focused to the CGCP with circular polarization. Since a portion of the light incident to the CGCP is reflected from coupler A and the half mirror, the focused spot is observed with the RLE and the CGCP by a microscope camera. Light radiated from coupler $\mathrm{C}$ is converted from rotation at a constant angular velocity to a linear uniform motion using an $\mathrm{f}-\theta$ lens. It is collimated by a relay lens and observed by an imaging camera. 
a

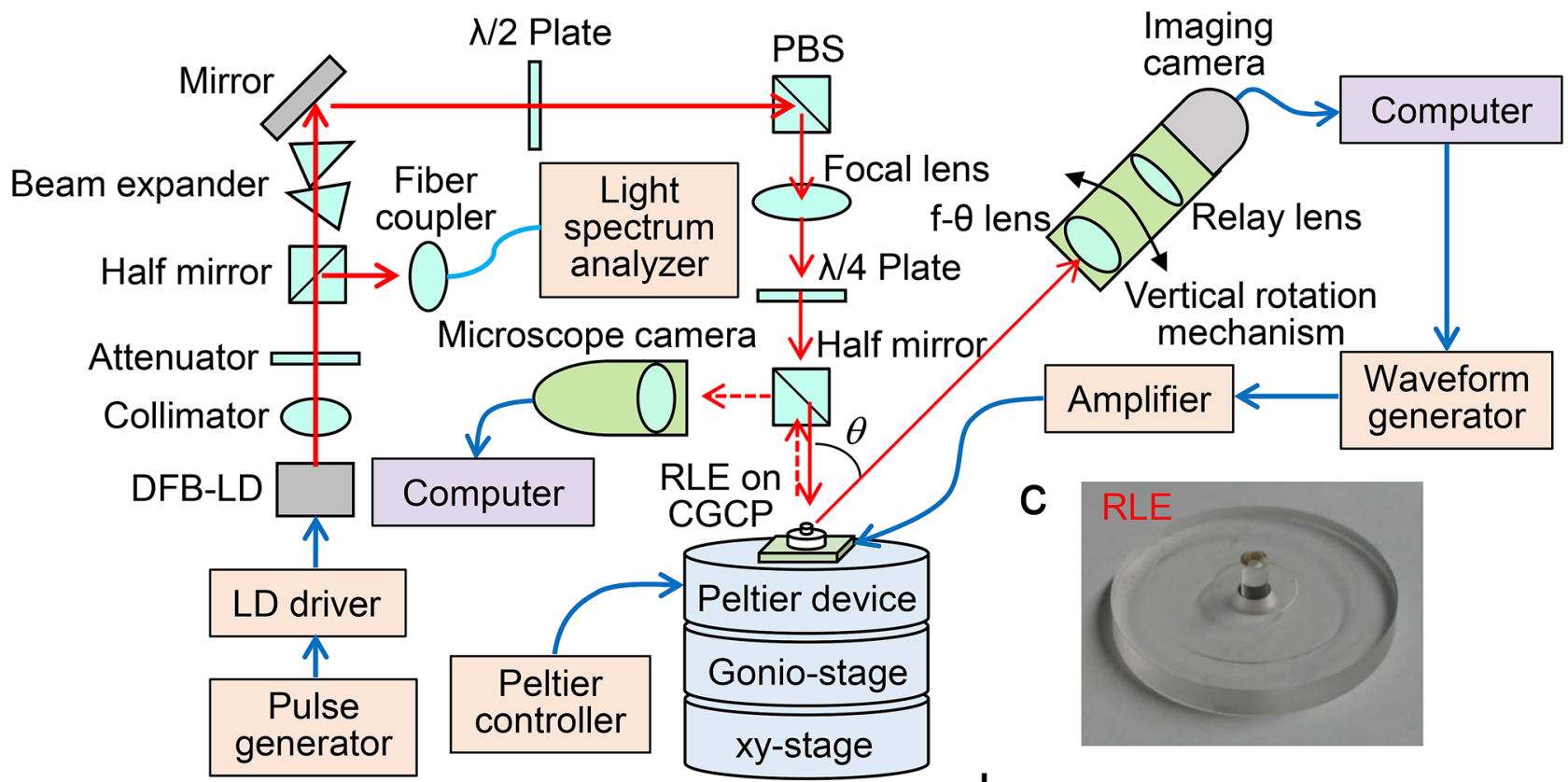

b

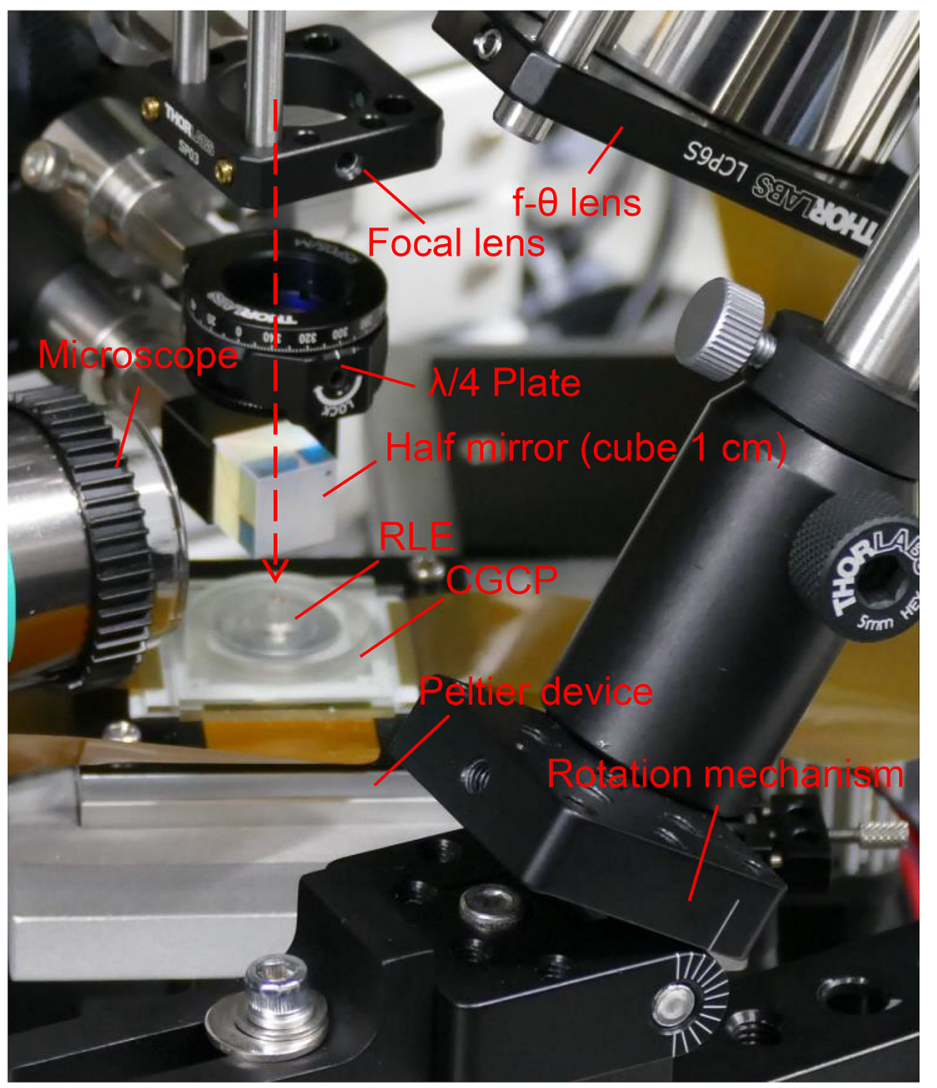

d

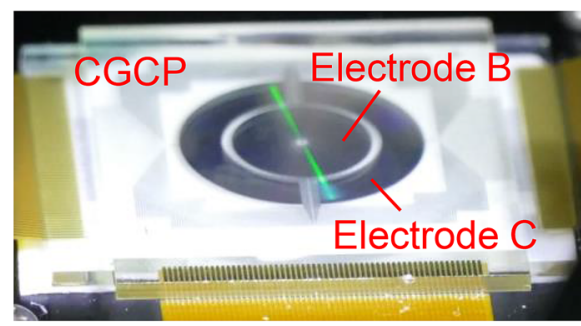

e

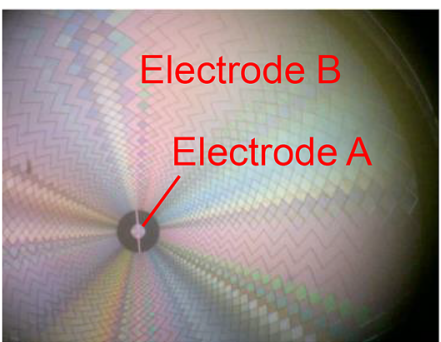

f

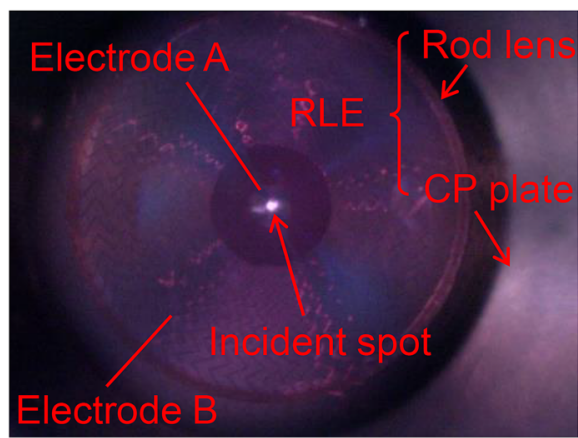

Figure 4. Experimental system for observation of scanning beams. (a) Configuration diagram of the experimental system. Light radiated from coupler $\mathrm{C}$ is converted from rotation at a constant angular velocity to a linear uniform motion by an $\mathrm{f}-\theta$ lens and is observed using an imaging camera. (b-d) Perspective photographs of a portion of the experimental system, the RLE, and the CGCP. (e) Photograph for a central surface of the CGCP under LC control, with RLE removed. (f) Observation photograph of a light spot incident to the surface of the CGCP using a microscope. 
Control signals applied to the electrodes of the CGCP are produced in computer-controlled waveform generators and are magnified by an amplifier. Figure 4e is a photograph of a central surface of the CGCP under LC control, with the RLE removed. Since the refractive indexes of the LC layer change under LC control, the external appearance is rotated according to the rotation of the diamond shapes in the radial direction (see Supplementary movie M1). Figure $4 \mathrm{f}$ shows an observation photograph of a light spot incident to the surface of the CGCP using a microscope. When the spot is adjusted at the center of coupler $\mathrm{A}$, the radiation from coupler $\mathrm{C}$ is confirmed by the imaging camera attached to the $\mathrm{f}-\theta$ lens.

\section{Experimental results}

Generally, although conventional LC devices including displays are not affected by the frequency of AC voltage, our device is affected. This is because while conventional devices exploit the change in alignment of the LC molecules across the whole layer thickness, our device exploits the change near the boundary face (i.e., in the evanescent field of the guided light). While the LC molecules tilt at an angle according to the electric field intensity, they continuously ramp, near the boundary face, from angle zero to the angle mentioned above (see Supplementary information S5). This boundary molecules are strongly affected by the frequency of AC voltage.

The voltage $V_{A}$ for electrode $A$ was set here to zero, because the amount of light radiated from coupler $C$ reached its maximum for $\mathrm{V}_{\mathrm{A}}=0$. This departure from the adjustable range may be due to a thickness error of $\mathrm{t}_{1}$ (i.e., $0.140 \mu \mathrm{m}$ designed vs. $0.132 \mu \mathrm{m}$ measured).

Figure $5 \mathrm{a}$ is an observation photograph indicating oriented states of the $\mathrm{LC}$ at the fringe region of the electrodes $B$ and $C$, where electrode $B$ is subject to a static state of $m=10$ and $V_{B}=3.2-40.0 \mathrm{~V}$ (distributed symmetrically about the $\mathrm{x}$-axis) with $\mathrm{t}_{\mathrm{B}}=1.0 \mathrm{~ms}$. As seen in Fig. $5 \mathrm{a}$, some disclinations are seen at the middle voltage regions, resembling soap bubbles, along the etched lines of the ITO layer (see Supplementary information S5 and S9).

Figure $5 \mathrm{~b}$ shows the relationship between the amount of light radiated from the coupler $\mathrm{C}$ after passing through the region of electrode $B$ and the constant voltage $V_{B}$ applied to the electrodes $B_{k}$ with the cycle $t_{B}$ of triangular $A C$ voltage as the parameter. When the cycle $t_{B}$ was made longer than $2 \mathrm{~ms}$, the observed horizontal spread angle of the radiated light increased gradually, expanding infinitely at over $10 \mathrm{~ms}$. Whereas, when the cycle $t_{B}$ was reduced to the range of $1 / 4-2.0 \mathrm{~ms}$, while the spread angle became smaller, the amount of the radiated light declined and disappeared up to $0.1 \mathrm{~ms}$.

Thus, the cycle conditions were set here as $\mathrm{t}_{\mathrm{B}}=1 \mathrm{~ms}$, and the periodic number is confined to $\mathrm{m}=10$, instead of $\mathrm{m}=5$, to increase a deflective power generated by the electrode B (see Supplementary information S10).

Vertical sweeping is caused by the change in ERI for the coupler $\mathrm{C}$ based on the formula of Eq. 1. Figure $5 c 1-c 3$ show observation photographs of vertically sweeping beams radiated from the coupler C (corresponding to the area just visible in Fig. 5a) using the imaging camera of the $\mathrm{f}-\theta$ lens (see supplementary movie M2). Their spots are generated by vibrating control of $\mathrm{t}_{\mathrm{C}}=1 / 32 \mathrm{~ms}$ and $\mathrm{V}_{\mathrm{C}}=0-20.0 \mathrm{~V}$ for electrode $\mathrm{C}$.

The amount of light rises and falls between half of a sweep cycle: this can be explained by using the result of Fig. 3f, where the states of Fig. $5 \mathrm{c} 1-\mathrm{c} 3$ correspond to $\mathrm{n}_{\mathrm{L}} \geq 1.65, \approx 1.62$, and $\leq 1.59$ for the curve of $\lambda=0.94 \mu \mathrm{m}$, respectively. In Fig. $5 \mathrm{cl}, \mathrm{c} 3$, the guided light passes through the region of coupler $\mathrm{C}$ before being totally radiated because the fabricated groove depth $(0.013 \mu \mathrm{m})$ was smaller than the designed value $(0.02 \mu \mathrm{m})$. The horizontal collimation is slightly changed with changes in the radiation angle, but it can be compensated by controlling the envelope shapes of the signals applied to $B_{k}$ according to the voltage of $C$.

Figure $5 \mathrm{~d}$,e show the vertical-steering performance for the voltage $\mathrm{V}_{\mathrm{C}}$ applied to the electrode $\mathrm{C}$ with the cycle $t_{C}$ of rectangular AC voltage as a parameter. They are measured under the same area shown in Fig. 5a. In Fig. $5 \mathrm{~d}$, the vertical displacement (or the sensitivity of vertical motion) peaks in the range of $\mathrm{t}_{\mathrm{C}}=1 / 8-1 / 32 \mathrm{~ms}$ and becomes about 10 degrees for the applied voltage of $20 \mathrm{~V}$. (The displacement for $t_{C}=1 \mathrm{~ms}$ is half of that for $t_{C}=1 / 8-1 / 32$ ms. Similarly, the maximum value of the refractive effect produced by the $B_{k}$ electrodes for $t_{B}=1 \mathrm{~ms}$ will be also reduced by half. This is the reason that $\mathrm{m}=10$ was selected in this experiment.) As shown in Fig. 5e, the horizontal spread angles (FWHM of spread angle) are stable within $0.3^{\circ}$ in the range equal to or less than $1 / 16 \mathrm{~ms}$, and the vertical ones are stable within $0.6^{\circ}-0.8^{\circ}$ for any cycle range.

Figure $5 \mathrm{f}$ shows the frequency response of the vertical displacement. We can see that the half maximum of the displacement amplitude is maintained up to $100 \mathrm{~Hz}$.

Basically, a frequency response of LC for electrode B has the same as that for electrode $\mathrm{C}$ which is shown in Fig. $5 \mathrm{~d}$. The reason why the results of Fig. $5 \mathrm{~b}$ show a faster damping for applied voltage and AC frequency, is affection by the disclinations shown in Fig. 5a. Although measuring the frequency response of the electrode $\mathrm{C}$ can be protected from affection by disclinations (i.e., by using lower frequency and lower voltage of the electrode B), measuring that of the electrode B is directly affected because guided light definitely passes through many points where disclinations are generated. The guided light is perturbed irregularly around the disclination line, and this is thought to be the cause that the light decays with higher frequency and higher voltage as shown in Fig. 5b.

Figure $5 \mathrm{~g}$ shows rotational sweeps in the rotational state of $\mathrm{T}_{\mathrm{B}}=0.5 \mathrm{~s}$ for electrode $\mathrm{B}$ and the static state of $\mathrm{t}_{\mathrm{C}}=1 / 16 \mathrm{~ms}$ and $\mathrm{V}_{\mathrm{C}}=15.0 \mathrm{~V}$ for electrode $\mathrm{C}$ (see Supplementary information S7and movie M3).

Figure $5 \mathrm{~h}$ shows image-analyzed results of a movie according to Fig. $5 \mathrm{~g}$. The amount of light is measured while stationary along the vertical line (according to argument $\varphi=0^{\circ}$ of Fig. $5 \mathrm{~g}$ or the $\mathrm{x}$-axis direction of Fig. 5a) of 6 pixels' width from each image. The amount of light rises towards a peak over a cycle of $36^{\circ}$. The variation of the peaks is due to the shortage of sampling numbers, which is a function of the frame rate of the image sensor. Figure $5 \mathrm{i}$ shows relationships between the spread angle and the rotating angle $\varphi$ corresponding to the spots shown in Fig. 5g. As shown in Fig. 5i, vertical spreads settle within $0.2^{\circ}-0.3^{\circ}$ and horizontal spreads vary over a 6 -degree cycle (i.e., the divided angle of electrode $\mathrm{B}_{\mathrm{k}}$ ) at $0.8^{\circ}-3.5^{\circ}$. This cycle change is caused by a deficit of phase correction for a single beam, because only three electrode lines per single beam can contribute to beam-collection as 

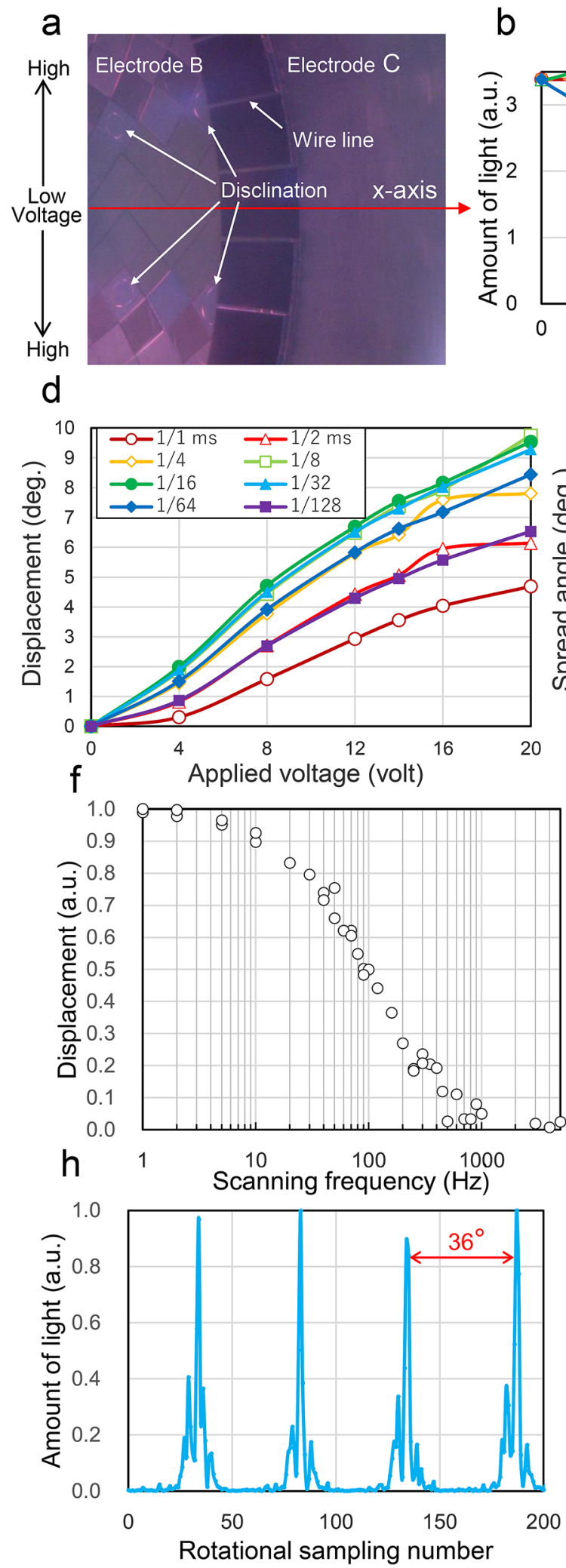
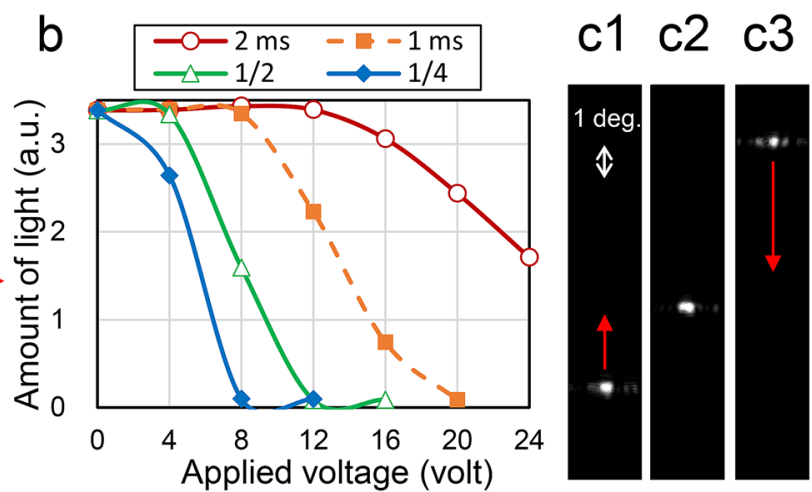

e

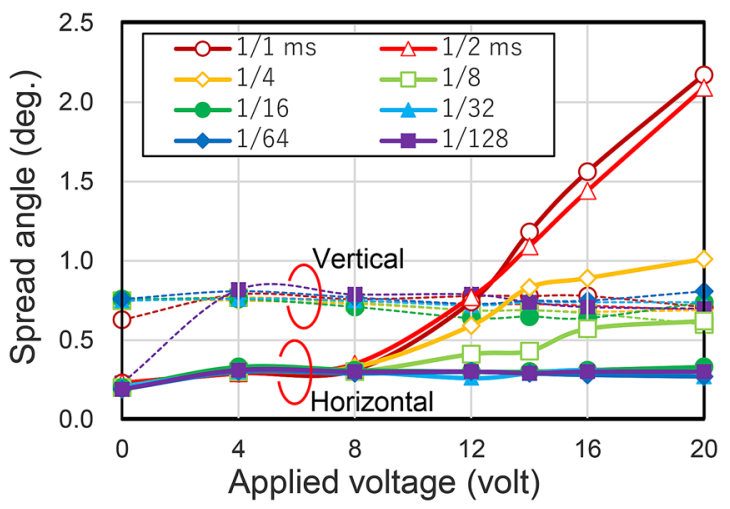

g
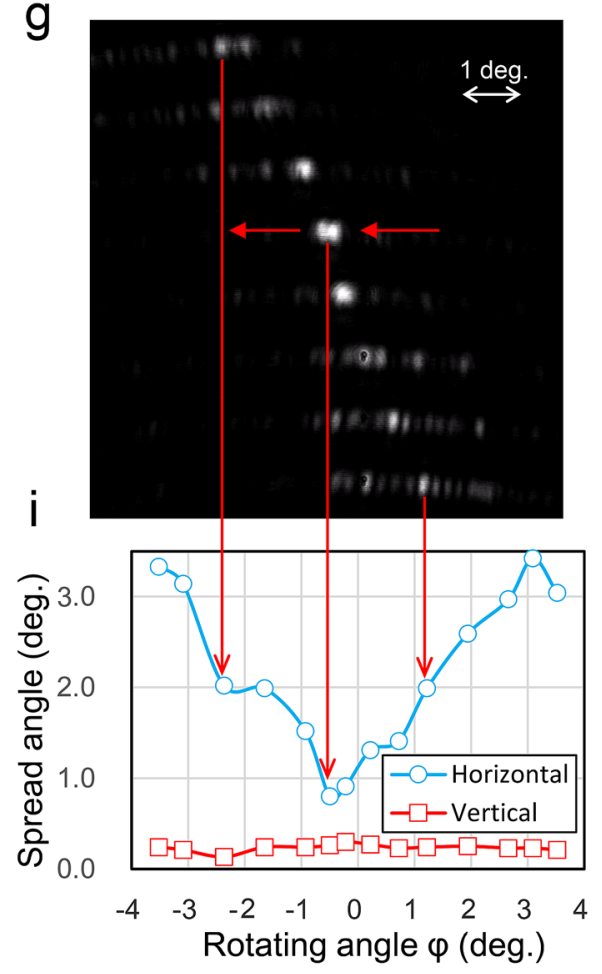
4Figure 5. Observation results. (a) Observation photograph indicating oriented states of the LC at the fringe region of the electrodes $B$ and $C$. Electrode $B$ is subject to a static state of $m=10$ and $V_{B}=3.2-40.0 \mathrm{~V}$ with $t_{B}=1.0 \mathrm{~ms}$. (b) Relationship between the amount of light radiated from the coupler $C$ and the constant voltage applied to the electrodes $B_{k}$ with the cycle $t_{B}$ of triangular $A C$ voltage as the parameter. (c1-c3) Observation photographs of vertically sweeping beams radiated from coupler $\mathrm{C}$ using the imaging camera of the $\mathrm{f}-\theta$ lens. The beams sweep by a vibrating control of $T_{C}=1.0 \mathrm{~s}$ and $V_{C}=0-20.0 \mathrm{~V}$ for electrode $C$. (d, e) Displacement and spread angles of vertical steering beams for the voltage applied to the electrode $C$ with the cycle $t_{C}$ of rectangular AC voltage as a parameter. The Peltier device is set at $26^{\circ} \mathrm{C}$. (f) Frequency response of the vertical displacement. Half maximum of the displacement amplitude is maintained up to $100 \mathrm{~Hz}$. (g) Observation photograph of rotationally sweeping beams radiated from coupler $\mathrm{C}$ using the imaging camera of the $\mathrm{f}-\theta$ lens. The beams show rotational sweeps by the rotational state of $T_{B}=0.5 \mathrm{~s}$ for electrode $\mathrm{B}$ and the static state of $\mathrm{t}_{\mathrm{C}}=1 / 16 \mathrm{~ms}$ and $\mathrm{V}_{\mathrm{C}}=15.0 \mathrm{~V}$ for electrode $\mathrm{C}$. (h) Image-analyzed results of a movie, showing amount of light. The beams sweep rotationally 10 times per turn. (i) Relationships between the spread angle and the rotating angle. Beams scan rotationally with a 6 -degree cycle variation of horizontal spread angle of between $0.8^{\circ}$ and $3.5^{\circ}$.

shown in Fig. 5a. The change could therefore be improved by, after overcoming problems such as disclinations, changing the periodic number from $m=10$ to $m=5$ or increasing the division number of electrode $B$.

\section{Conclusion}

We have demonstrated the world's first two-axis scanner using liquid crystal control in which the scanning ranges were 360 degrees in the horizontal direction and 10 degrees in the vertical direction: the beam of a spread angle of $0.3^{\circ} \times 0.8^{\circ}$ at minimum sweeps vertically up to $100 \mathrm{~Hz}$ frequency and ten equally-spaced beams scan rotationally with a 6-degree cycle variation of spread angle of between $0.8^{\circ}$ and $3.5^{\circ}$.

The performances of spread angles and motion ranges can be improved by dealing with challenges like the disclinations at high voltages. The sweep frequency can be also improved by changing the thickness of the LC layer from $5 \mu \mathrm{m}$ to less than $0.5 \mu \mathrm{m}$ (see Supplementary information S11), since our device exploits the change at the surface layer interface of the LC. Because the frequency response of the LC is inversely proportional to the square of the thickness, a sweep frequency of more than $10 \mathrm{kHz}$ (or $50 \mathrm{kHz}$ if we add the five-beams effect) can be anticipated.

The input efficiency of coupler A for incident light, which is circularly polarized, falls to $40-50 \%$ because only TE mode light can be excited, and this coupled light can be fully radiated only from coupler $\mathrm{C}$. The input efficiency of coupler $\mathrm{C}$ for return light, which is randomly polarized, also falls to $40-50 \%$ because only TE mode light can be excited. In the experiment, these efficiencies were thought not to be sufficient because the groove depth was shallower than the designed value. If a PBS and a half mirror are added to the system, outward efficiency will decrease by half (i.e., to $20-25 \%$ ) and return efficiency will fall by $3 / 4$ (to $30-37.5 \%$ ).

Although production errors in as refractive indexes, wavelength, and layer thicknesses, etc., affect input coupling at coupler A and radiation at coupler $\mathrm{C}$, these effects are to some extent counteracted by the LC control (see Supplementary information S12).

Some mechanical LIDARs originally have a 360-degree scanning range in the horizontal direction and their detection structures are used concomitantly with the emission structures. However, all conventional SS-LIDARs significantly narrow their ranges of motion to enable non-mechanical scanning, and most of them (except for MEMS-LIDARs) require detection structures that are separate from their emission structures.

Our CGC-LIDAR maintains a 360-degree scanning range and the detection and emission structures can be combined. Moreover, it produces multi-sweep beams that provide a higher-definition image enabling the "level 5 ," and the S/N ratio of TOF signals detected using our method is anticipated to be 50 -fold that achieved by conventional methods using a band pass filter due to the effect of the wavelength selectivity of the coupler (see Supplementary information S13).

We believe our method closely matches the requirements of SS-LIDAR. We will aim in future to achieve better performance and will at the same time investigate the potential for a low-cost mass-fabrication process.

Liquid crystal technology has seen considerable success in many areas. We anticipate that our experiments in LIDAR will open a new and expanding field for its use.

\section{Materials and methods}

Construction materials. The rod lens and CP plate are made of S-BSM14 by Ohara. The HR plate is made of BOC30 by Sumita. For the liquid crystal, 5CB (4-cyano-4'-pentylbiphenyl by TCI) is used.

Measuring equipment. Light emitted from a DFB-LD (EYP-DFB-0935 by Eagleyard) is collimated and shaped into a parallel beam with circular spread by a collimator (C230TMD-B mounted on LDH3-P1/M by Thorlabs) and a beam expander (anamorphic prism pairs \#47-274 by Edmund). After a portion of light is split by a half mirror (Non-Polarizing Beam splitter CCM5-BS017/M by Thorlabs) and coupled by a fiber coupler (fiber port PAF2P-15B by Thorlabs), the light's wavelength is monitored by a light spectrum analyzer. The beam transmitted by the half mirror is adjusted by rotation of a $\lambda / 2$ plate to minimize transmission through a polarization beam splitter (PBS, CCM5-PBS202 by Thorlabs). The light reflected from the PBS is circularly polarized by a $\lambda / 4$ plate and is vertically focused to a CGCP by a focal lens $(\mathrm{f}=100 \mathrm{~mm})$. Since a portion of the incident light is reflected from coupler A and the half mirror (BS011 by Thorlabs), the focused spot is observed with the RLE and the CGCP using a microscope camera (DINOAM7915MZTL by Dino). The CGCP is located on a Peltier device (VPE20-30S by VICS) and is thermally controlled at $26^{\circ} \mathrm{C}$. The Peltier device is also located on the Gonio-stages 
and $\mathrm{x}$ - and $\mathrm{y}$-stages for positioning. Light radiated from coupler $\mathrm{C}$ is converted by an $\mathrm{f}-\theta$ lens (LSM05-BB by Thorlabs), collimated by a relay lens (LB1723-B by Thorlabs) and observed by an imaging camera that includes an image sensor (CM3-U3-13Y3M-CS by FLIR). The $\mathrm{f}-\theta$ lens unit is set up on a vertically rotating mechanism. Voltage signals applied to the electrodes of the CGCP are produced in waveform generators (AWG-10 by Elmos) controlled by a computer and are magnified by up to fourfold by an amplifier.

Estimation of spread angles. Spread angle is estimated by calculating the standard deviation of an intensity distribution along the vertical or horizontal cross-section passing through a peak point driven from jpeg images. The intensity is expressed by an integer value from 0 to 255: some of the lower range can be cut off as noise. Since the cutoff level affects the calculated result of the spread angle, it must be carefully identified and selected. The level is set here at 3\% (the level for a black background) of 255 .

Received: 18 March 2021; Accepted: 30 June 2021

Published online: 20 July 2021

\section{References}

1. Wyant, J. C. Rotating diffraction grating laser beam scanner. Appl. Opt. 14, 1057-1058 (1975).

2. Matsuda, T., Abe, F. \& Takahashi, H. Laser printer scanning system with a parabolic mirror. Appl. Opt. 17, 878-884 (1978).

3. Varughese, K. O. G. \& Krishna, K. S. R. Flattening the field of post-objective scanners by optimum choice and positioning of polygons. Appl. Opt. 32, 1104-1108 (1993).

4. Asada, N., Matsuki, H., Minami, K. \& Esashi, M. Silicon micromachined two-dimensional galvano optical scanner. IEEE Trans. Mag. 30, 4647-4649 (1994).

5. Miyajima, H. et al. A MEMS electromagnetic optical scanner for a commercial confocal laser scanning microscope. J. Microelectron. Syst. 12, 243-251 (2003).

6. Yalcinkaya, A. D., Urey, H., Brown, D., Montague, T. \& Sprague, R. Two-axis electromagnetic microscanner for high resolution displays. J. Microelectron. Syst. 15, 786-794 (2006).

7. Chellappan, K. V., Erden, E. \& Urey, H. Laser-based displays: a review. Appl. Opt. 49, F79-F98 (2010).

8. Meyer, R. A. Optical beam steering using a multichannel lithium tantalate crystal. Appl. Opt. 11, 613-616 (1972).

9. Resler, D. P., Hobbs, D. S., Sharp, R. C., Friedman, L. J. \& Dorschner, T. A. High-efficiency liquid-crystal optical phased-array beam steering. Opt. Lett. 21, 689-691 (1996).

10. Thomas, J. A. \& Fainman, Y. Optimal cascade operation of optical phased-array beam deflectors. Appl. Opt. 37, 6196-6212 (1998).

11. Xiao, F., Hu, W. \& Xu, A. Optical phased-array beam steering controlled by wavelength. Appl. Opt. 44, 5429-5433 (2005).

12. Acoleyen, K. V. et al. Off-chip beam steering with a one-dimensional optical phased array on silicon-on-insulator. Opt. Lett. 34, 1477-1479 (2009).

13. Acoleyen, K. V., Rogier, H. \& Baets, R. Two-dimensional optical phased array antenna on silicon-on-insulator. Opt. Exp. 18, $13655-13660$ (2010).

14. Kwong, D., Hosseini, A., Zhang, Y. \& Chenb, R. T. $1 \times 12$ Unequally spaced waveguide array for actively tuned optical phased array on a silicon nanomembrane. Appl. Phys. Lett. 99, 051104 (2011).

15. Doylend, J. K. et al. Two-dimensional free-space beam steering with an optical phased array on silicon-on-insulator. Opt. Exp. 19, 21595-21604 (2011).

16. Sun, J., Timurdogan, E., Yaacobi, A., Hosseini, E. S. \& Watts, M. R. Large-scale nanophotonic phased array. Nature 493, 195-199 (2013).

17. Gu, X., Shimada, T. \& Koyama, F. Giant and high-resolution beam steering using slow-light waveguide amplifier. Opt. Exp. 19, 22675-22683 (2011).

18. Gu, X., Shimada, T., Matsutani, A. \& Koyama, F. Ultra-high channel-count wavelength demultiplexer based on a Bragg reflector waveguide with large angular dispersion. Opt. Exp. 20, B331-B338 (2012).

19. Ito, H. et al. Wide beam steering by slow-light waveguide gratings and a prism lens. Optica 7, 47-52 (2020).

20. Scrymgeour, D. A. et al. Large-angle electro-optic laser scanner on LiTaO3 fabricated by in situ monitoring of ferroelectric-domain micropatterning. Appl. Opt. 40, 6236-6241 (2001).

21. Nakamura, K., Miyazu, J., Sasaura, M. \& Fujiura, K. Wide-angle, low-voltage electro-optic beam deflection based on space-chargecontrolled mode of electrical conduction in $\mathrm{KTa}_{1-x} \mathrm{Nb}_{x} \mathrm{O}_{3}$. Appl. Phys. Lett. 89, 131115 (2006).

22. Kurosaka, Y. et al. On-chip beam-steering photonic-crystal lasers. Nat. Photo. 4, 447-450 (2010).

23. Davis, S. R., Farca, F., Rommel, S. D., Martin, A. W. \& Anderson, M. H. Analog, non-mechanical beam-steerer with 80 degree field of regard. Proc. SPIE 6971, 69710G (2008).

24. Nishiwaki, S., Asada, J. \& Uchida, S. Optical head employing concentric-circular focusing grating coupler. Appl. Opt. 33, 1819-1827 (1994).

25. Nishiwaki, S. Aberrations and convergence characteristics of a concentric-circular focusing grating coupler. Appl. Opt. 34, 73617371 (1995).

26. Nishiwaki, S., Asada, J., Ooshima, K. \& Kitagawa, T. Fabrication of a concentric-circular focusing grating coupler utilizing a conicwavefront interference method, and light convergence experiments using the coupler. Appl. Opt. 34, 7372-7382 (1995).

27. Tamir, T. \& Peng, S. T. Analysis and design of grating couplers. Appl. Phys. 14, 235-254 (1977).

\section{Acknowledgements}

The author thanks Y. Inoue for the initial incentive and T. Korenaga and T. Hirasawa for providing financial support for the development of devices.

\section{Author contributions}

S. N. devised the concept, designed and analyzed the experimental sample. He also established the experiment and evaluation system, carried out assessment experiments and monitored progress.

\section{Competing interests}

The author declares no competing interests. 


\section{Additional information}

Supplementary Information The online version contains supplementary material available at https://doi.org/ 10.1038/s41598-021-94208-2.

Correspondence and requests for materials should be addressed to S.N.

Reprints and permissions information is available at www.nature.com/reprints.

Publisher's note Springer Nature remains neutral with regard to jurisdictional claims in published maps and institutional affiliations.

(c) (i) Open Access This article is licensed under a Creative Commons Attribution 4.0 International License, which permits use, sharing, adaptation, distribution and reproduction in any medium or format, as long as you give appropriate credit to the original author(s) and the source, provide a link to the Creative Commons licence, and indicate if changes were made. The images or other third party material in this article are included in the article's Creative Commons licence, unless indicated otherwise in a credit line to the material. If material is not included in the article's Creative Commons licence and your intended use is not permitted by statutory regulation or exceeds the permitted use, you will need to obtain permission directly from the copyright holder. To view a copy of this licence, visit http://creativecommons.org/licenses/by/4.0/.

(C) The Author(s) 2021 